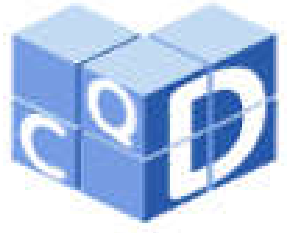

Revista Eletrônica Paulista de Matemática

ISSN 2316-9664

Volume 14, fev. 2019

Edição Ermac

Sérgio Luís Tamássia dos Santos

USP - Universidade de São

Paulo

sltsantos@usp.br

Helton Hideraldo Bíscaro

USP - Universidade de São

Paulo

heltonhb@usp.br

\section{Revisão sistemática sobre a utilização de jogos sérios na aprendizagem de matemática}

Systematic review of the use of serious games in math learning

\section{Resumo}

As mudanças que as inovações tecnológicas têm promovido em nossas vidas estão cada vez mais profundas e rápidas. As crianças de hoje são nativos digitais, utilizando videogames, smartphones e computadores, por vezes até antes de sua alfabetização. A educação, uma das bases que propiciou esta revolução, e, de uma forma mais específica o ensino de matemática, essencial no campo de ciências e engenharia, necessita acompanhar estas mudanças, com a adoção de novas tecnologias em sala de aula. Este trabalho tem por objetivo buscar uma resposta para uma das questões levantadas por este cenário: qual a real efetividade do uso de jogos sérios eletrônicos no processo de ensinoaprendizagem de matemática na educação básica? Através de uma revisão sistemática apresentamos um panorama do estudo acadêmico sobre o tema nos últimos cinco anos, concluindo que realmente os jogos sérios eletrônicos são ferramentas de alavancagem no processo de ensino-aprendizagem de matemática.

Palavras-chave: Matemática. Ensino. Jogos sérios. Revisão sistemática.

\begin{abstract}
Technological innovations have promoted changes in our lives, in a way ever deeper and faster. Today's kids are digital natives, playing video games, using smartphones, and computers sometimes even before their literacy. Education, which is one of the foundations for this revolution, and, more specifically, the teaching of mathematics, which is essential in the field of science and engineering, has the need to follow these changes through the adoption of new technologies in classroom. This paper aims to find an answer to one of the questions raised by this scenario: what is the real effectiveness of the use of serious electronic games in the teaching-learning process of mathematics in basic education? Through a systematic review we present a panorama of the academic study on the subject in the last five years, concluding that serious electronic games are actually powerful tools in the teaching-learning process of mathematics.

Keywords: Mathematics, basic education, serious game, gamification, systematic review.
\end{abstract}




\section{Introdução}

Todos nós já nos deparamos com jogos eletrônicos em computadores, consoles e dispositivos móveis. Desde o final do século XX nossos lares têm sido invadidos, com o advento da revolução digital que vivemos. Palfrey e Gasser (2011) afirmam que "o mais incrível (..) é a maneira em que a era digital transformou o modo como as pessoas vivem e se relacionam umas com as outras e com o mundo que as cerca.".

A geração que tem ocupado os bancos escolares atualmente, nasceu já dentro desta revolução, gerando um conflito entre a forma de educação encontrada nas salas de aulas e o estilo de aprendizagem que ela espera. Segundo Beck e Wade (2006) eles preferem se auto educar, utilizando o erro como forma de aprendizado para novas tentativas, interagindo fortemente com seus pares, tendo a prática como forma preponderante ao invés do uso de livros e manuais.

Essa mudança de comportamento faz com que temas como jogos sérios ou serious games na educação tomem corpo no meio acadêmico. Segundo a definição de Sawyer (2002 apud DJAOUTI; ALVAREZ; JESSEL, 2011) de uma forma geral como um programa de computador que mistura um uso não de entretenimento ("serious") com uma estrutura de videogame ("games"), ou "gamificação" ou Gamification, que segundo Deterding (2011) é a utilização de elementos de "design" de jogos em contextos não relacionados a jogos.

O objetivo deste artigo é buscar respostas sobre o impacto do uso de jogos sérios eletrônicos como auxiliares no processo de ensino-aprendizagem de matemática na educação básica. Para atingi-lo, realizamos uma revisão sistemática da literatura existente sobre o tema, tomando por base o artigo de Tamássia dos Santos e Biscaro (2018), apresentado no V ERMAC realizado pela Universidade Estadual Paulista "Júlio de Mesquita Filho" entre os dias 6 e 8 de junho de 2018 em Bauru, SP, Brasil.

Buscamos discutir com mais profundidade o processo de revisão sistemática, inclusive com a apresentação de um modelo, além de acrescentar mais dados relativos aos resultados obtidos, expandindo suas conclusões, realizando uma avaliação de seus benefícios, dificuldades e potenciais problemas dentro do conjunto de estudos acadêmicos sobre o tema nos últimos cinco anos.

Este artigo está dividido da seguinte forma: além desta introdução, que procura contextualizar o problema e estabelecer os objetivos desta pesquisa; a sessão 2 introduz os conceitos básicos de uma revisão sistemática de literatura e estabelece os procedimentos metodológicos utilizados nesta pesquisa. Na sessão 3 são apresentados os resultados da condução da visão e finalmente na sessão 4 as discussões e conclusões baseadas nos resultados são apresentadas.

\section{A revisão sistemática}

Segundo Kitchenham (2004) "uma revisão sistemática de literatura é um meio de identificar, avaliar e interpretar toda produção de pesquisa disponível para uma particular questão que se está pesquisando, ou para uma determinada área de pesquisa ou para um fenômeno de interesse."

Existem várias razões para se realizar uma revisão sistemática, entre as quais podemos citar:

- Reunir evidências que dizem respeito a um determinado assunto ou tecnologia;

- Identificar lacunas na pesquisa atual, com a descoberta de novas áreas de investigação;

- Fornecer embasamento/estrutura para o posicionamento adequado de novas atividades de pesquisa (KITCHENHAM, 2004).

$\mathrm{Na}$ maioria das introduções e seções teóricas de trabalhos científicos existem revisões de literatura. O grande problema em uma revisão é que somente os principais estudos em

SANTOS, S. L. T. dos; BÍSCARO, H. H. Revisão sistemática sobre a utilização de jogos sérios na aprendizagem de matemática. C.Q.D.- Revista Eletrônica 
determinada área são incluídos, ou ainda, em um viés tendencioso, apenas aqueles que são aderentes à opinião pessoal do conjunto de autores, ou aos resultados esperados (NIGHTINGALE, 2009).

Excluir estudos menores, pode ocultar resultados relevantes que serão desconsiderados. Nightingale (2009) cita uma publicação de Lau et al (2002 apud NIGHTINGALE, 2009) onde foi realizada uma meta análise cumulativa sobre o uso de uma terapia trombolítica em pacientes infartados. Dentre os pontos encontrados haviam diversos estudos com resultados importantes sobre a redução de mortalidade realizados em 1973. Mesmo assim, 13 anos depois, em 1986 esse procedimento não havia sido implementado rotineiramente na prática clínica. Dentre as razões, uma delas estava relacionada a não existir relevância estatística para estes estudos isoladamente, porém ao combiná-los usando técnicas de meta análise, foi possível detectar o efeito protetor dessa terapia.

O valor científico de uma revisão sistemática está baseado em sua completude e no caráter justo de seleção. A forma de obter isto é conduzi-las utilizando uma estratégia de pesquisa previamente definida, incluindo seus termos no resultado da revisão em questão.

Esta estratégia deve permitir avaliar o quão completa e justa é a revisão, incluindo resultados que apoiem as hipóteses, da mesma forma que aqueles que não as apoiem (KITCHENHAM, 2004).

Para atingir os resultados, alguns pontos necessitam ser claramente definidos, normalmente em um instrumento conhecido como protocolo da revisão, que geralmente contém os seguintes itens:

- Os objetivos da revisão;

- Os critérios de inclusão e exclusão para estudos;

- A forma como os estudos serão identificados;

- O plano de análise.

As maiores dificuldades na execução de uma revisão sistemática residem no esforço de refinamento das metodologias de planejamento e execução, e na necessidade de muitas vezes reiniciar o processo devido a mudanças no planejamento.

\subsection{Ganhos e perdas}

A maior vantagem de uma revisão sistemática é que ela provê informações sobre os efeitos de certos fenômenos através de uma ampla gama de configurações e métodos de pesquisa, como técnicas de meta análise. Se há consistência nos dados dos estudos revisados são fornecidos resultados robustos, se há inconsistência, a fonte destas variações pode ser estudada. Porém há o risco de aumentar-se a relevância de pequenos desvios, tornando-os efeitos reais (KITCHENHAM, 2004).

De outro lado, a execução de uma revisão sistemática requer um esforço maior que uma revisão tradicional. Parte deste diz respeito à documentação que é gerada no processo de execução, pronta para utilização na pesquisa e, em sua publicação (NUNES, 2010).

A condução de uma revisão sistemática não é uma tarefa simples, sendo necessário agir de forma objetiva, transparente e rigorosa. É a elaboração de um protocolo de revisão fixo que guiará o trabalho aderente a estas características, além de permitir que haja replicabilidade em todas as avaliações (MALLETT et al, 2012). Dentre suas maiores dificuldades de execução está o esforço de refinamento das metodologias de planejamento e execução, e na necessidade de reiniciar o processo, devido a mudanças no plano de ação, para impedir resultados tendenciosos (BIOLCHINI et al, 2005).

\subsection{Processo de revisão sistemática}

SANTOS, S. L. T. dos; BÍSCARO, H. H. Revisão sistemática sobre a utilização de jogos sérios na aprendizagem de matemática. C.Q.D.- Revista Eletrônica Paulista de Matemática, Bauru, v. 14, p. 12-25, fev. 2019. Edição Ermac.

DOI: 10.21167/cqdvol14ermac201923169664sltshhb1225 Disponível em: https://www.fc.unesp.br/\#!/departamentos/matematica/revista-cqd/ 
Nunes (2010) apresenta um processo de revisão sistemática, dividido em fases de planejamento, revisão e documentação:

1. Planejamento.

a. Identificação da necessidade da revisão:

. Nesta etapa define-se o objetivo da revisão.

b. Desenvolvimento de um protocolo de revisão:

. Especifica o que se espera que seja respondido no final da revisão sistemática. Esta resposta pode ser de uma questão objetiva, ou mais subjetiva como o de uma análise exploratória dependendo do foco da revisão e de suas especificidades;

. Define as estratégias que serão utilizadas: palavras-chave, mecanismos de busca, delimitação do escopo;

. Estabelece os critérios de seleção de estudo, tanto de inclusão quanto de exclusão, assim como os de qualidade;

. Define a estratégia de seleção de dados e de síntese dos dados extraídos.

2. Condução da Revisão.

a. Identificação da pesquisa:

. Identificar revisões sistemáticas já existentes;

. Avaliar o volume de estudos potencialmente relevantes;

. Triar utilizando combinações de palavras-chave derivadas das questões formuladas;

. Revisão dos resultados da pesquisa;

. Definir bases de dados eletrônicos que serão pesquisadas;

. Consultar, se necessário, especialistas da área;

. Documentar a busca;

- Gerenciar a bibliografia.

b. Seleção de estudos primários:

. Primeira fase: seleção por títulos e sumários (abstracts);

- Obter cópia dos documentos selecionados na primeira fase;

. Segunda fase: avaliar texto completo;

. Manter uma lista dos excluídos, com motivos (critérios de inclusão e exclusão previamente definidos);

. Checar decisões de inclusão com todos os pesquisadores do grupo para obter confiabilidade no processo de seleção.

c. Avaliação da qualidade dos estudos:

. Realizar em grupo ou com o orientador;

. Refinar os critérios de inclusão e exclusão;

. Interpretar o material encontrado;

. Gerar recomendações para pesquisas adicionais.

d. Extração e monitoramento dos dados:

. Criar formulário de extração de dados, mantendo dados necessários para referência;

. Conteúdo: data, título, autores, fonte, observações;

. Consolidar dados para evitar repetições.

e. Síntese dos dados:

. Resumir os resultados dos estudos primários, preferencialmente de forma quantitativa, podendo ser descritiva;

. Síntese quantitativa: meta análise (análise estatística);

SANTOS, S. L. T. dos; BÍSCARO, H. H. Revisão sistemática sobre a utilização de jogos sérios na aprendizagem de matemática. C.Q.D.- Revista Eletrônica

Paulista de Matemática, Bauru, v. 14, p. 12-25 fev. 2019. Edição Ermac.

DOI: 10.21167/cqdvol14ermac201923169664sltshhb1225 Disponível em: https://www.fc.unesp.br/\#!/departamentos/matematica/revista-cqd/ 
. Apresentação dos resultados: gráficos, tabelas;

. Apresentação dos resultados: respostas às perguntas que originaram a revisão.

3. Documentação:

. Descrever em um relatório técnico ou artigo o processo, reflexões e conclusões.

A revisão sistemática é uma metodologia científica com uma grande importância, pelo seu potencial de integrar resultados de diversas pesquisas empíricas e, de reunir informações de determinado tema de forma estruturada e reproduzível.

Dada sua importância, a condução de revisões sistemáticas não é uma tarefa simples, pois envolve atividades complexas e, a compreensão de conceitos e termos que podem não ser conhecidos pelos pesquisadores.

A fase de execução é um ponto importante a ser abordado, pois a experiência tem mostrado que a pesquisa e avaliação dos estudos ainda representam um gargalo no processo de revisão sistemática (BIOLCHINI et al, 2005).

\section{Aplicação do processo de revisão sistemática}

Inicialmente elaboramos um protocolo de revisão, definindo um foco para a pergunta, sua qualidade e amplitude, e os conjuntos de palavras-chave e sinônimos (Tabela 1), de forma a abranger tanto, trabalhos que tratassem de "jogos sérios", quanto de uso de "gamificação", foram definidas duas strings para as buscas (Tabela 2).

Tabela 1 - Formulação da pergunta

\begin{tabular}{|c|l|}
\hline Foco da Pergunta & $\begin{array}{l}\text { A utilização de jogos eletrônico é efetiva no ensino de ma- } \\
\text { temática no ensino fundamental? }\end{array}$ \\
\hline $\begin{array}{c}\text { Qualidade e Amplitude } \\
\text { da Pergunta }\end{array}$ & $\begin{array}{l}\text { Existem várias utilizações de jogos não-eletrônicos na li- } \\
\text { teratura, assim como utilização de recursos de tecnologia } \\
\text { da informação que não são jogos. Existe a necessidade de } \\
\text { avaliar-se este quesito com critério de forma a não conta- } \\
\text { minar o resultado final. Serão analisadas publicações ci- } \\
\text { entíficas e livros indexados em meio eletrônico. }\end{array}$ \\
\hline Palavras-chave e sinônimos & $\begin{array}{l}\text { Matemática (mathematics), jogos sérios (serious game), } \\
\text { educação básica (basic education), "gamificação" (ga- } \\
\text { mification) }\end{array}$ \\
\hline
\end{tabular}

Tabela 2 - Strings de busca

\begin{tabular}{|l|l|}
\hline String \#1 & "serious game" "mathematics" "basic education" \\
\hline String \#2 & "gamification" "mathematics" "basic education" \\
\hline
\end{tabular}

Também foram selecionados os idiomas e as fontes utilizados e os critérios de inclusão e exclusão apresentados nas tabelas 3 e 4 respectivamente.

Tabela 3 - Seleção de fontes e idiomas

SANTOS, S. L. T. dos; BÍSCARO, H. H. Revisão sistemática sobre a utilização de jogos sérios na aprendizagem de matemática. C.Q.D.- Revista Eletrônica Paulista de Matemática, Bauru, v. 14, p. 12-25, fev. 2019. Edição Ermac.

DOI: 10.21167/cqdvol14ermac201923169664sltshhb1225 Disponível em: https://www.fc.unesp.br/\#!/departamentos/matematica/revista-cqd/ 


\begin{tabular}{|l|l|}
\hline Critério de seleção de fontes & $\begin{array}{l}\text { Abrangência e acessibilidade para que os resultados pos- } \\
\text { sam ser facilmente reproduzidos }\end{array}$ \\
\hline Lista de fontes & Google Scholar IEEE \\
\hline $\begin{array}{l}\text { Idiomas que serão pesquisa- } \\
\text { dos }\end{array}$ & Português, Inglês e Espanhol \\
\hline
\end{tabular}

Tabela 4 - Critérios de inclusão e de exclusão

\begin{tabular}{|l|l|}
\hline \multicolumn{2}{|c|}{ Critérios de inclusão } \\
\hline 1 & Jogos sérios (serious games) eletrônicos ou aplicações "gamificadas" \\
\hline 2 & Área de ensino de matemática \\
\hline 3 & Educação básica - no Brasil compreendendo Ensino Fundamental I e II \\
\hline 4 & Tecnologia de jogos computacionais \\
\hline 5 & Período de 2012 a 2017 \\
\hline 6 & Linguagens: Português, Inglês e Espanhol \\
\hline & \multicolumn{1}{c|}{ Critérios de exclusão } \\
\hline 1 & Jogos de entretenimento sem fins educativos \\
\hline 2 & Outras áreas de ensino que não matemática \\
\hline 3 & Ensinos médio, superior e pós-graduações \\
\hline 4 & Jogos não computacionais \\
\hline 5 & Período anterior a 2012 \\
\hline 6 & Outras linguagens que não Português, Inglês e Espanhol \\
\hline 7 & Artigos repetidos \\
\hline
\end{tabular}

Esta definição de palavras-chave ocorreu através de um processo de refinamento, buscando encontrar um número adequado de trabalhos para se submeter ao processo de revisão, que consistiu em uma primeira etapa, na seleção através dos sumários (abstracts), e posteriormente, na leitura integral dos trabalhos.

\subsection{Condução da revisão sistemática}

A tabela 5 apresenta um resumo do resultado das pesquisas na base do Google Scholar, na base Scielo e no IEEE, realizadas entre os dias 20 e 30 de abril de 2017 utilizando as palavras chaves "serious game" "mathematics" "basic education".

Tabela 5 - Resultados de buscas para "serious game" "mathematics" "basic education"

\begin{tabular}{|l|l|}
\hline Google Scholar & 44 \\
\hline Scielo & 1 \\
\hline IEEE & 6 \\
\hline Total de resultados & 51 \\
\hline Total de selecionados & 6 \\
\hline
\end{tabular}

Somente $11,76 \%$ dos resultados obtidos foram selecionados após a análise, utilizando-se os critérios definidos na tabela 4, em um total de 6 artigos/estudos.

Os critérios de exclusão mais utilizados foram:

- "3. Ensinos médio, superior e pós-graduações", com 70,59\%. 
- "2. Outras áreas de ensino que não matemática" com 64,71\%.

(Obs. diversos artigos foram eliminados por mais de um critério, a soma das porcentagens não é $100 \%$ ). Os demais critérios tiveram participações menores do que 5\% cada um. A Figura 1 mostra os resultados:

Figura 1 - Critérios de exclusão mais utilizados para a busca "serious game" "mathematics" "basic education"

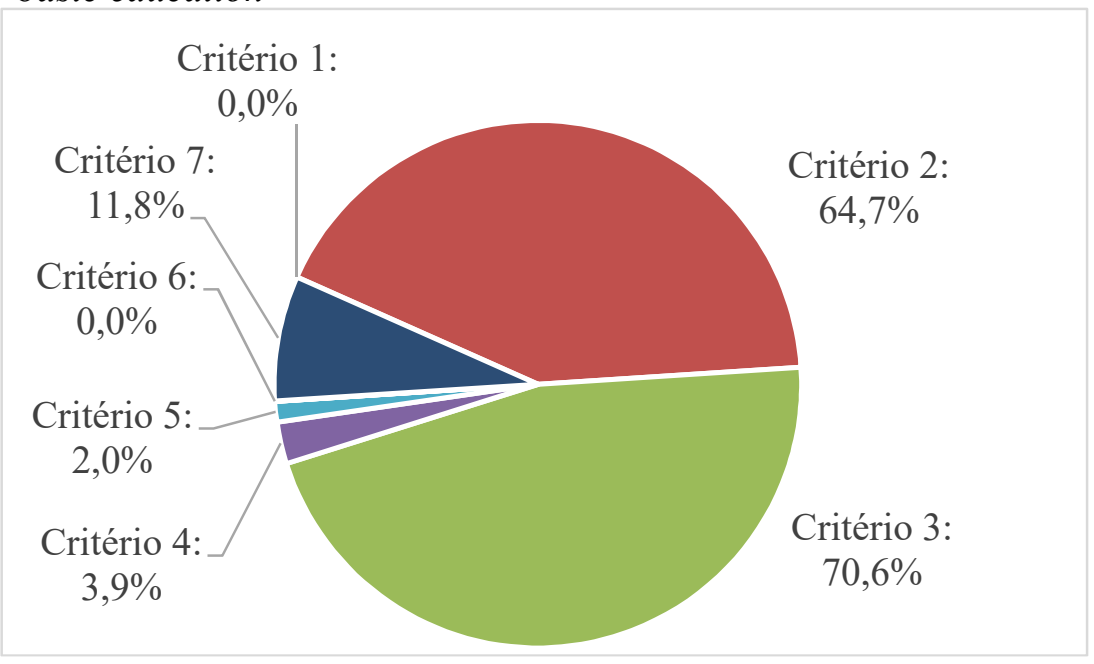

Utilizando-se a string "gamification" "mathematics" "basic education" foi realizada a segunda etapa da pesquisa. Dos 145 artigos pesquisados através do Google Scholar, Scielo e IEEE entre os dias 20 e 30 de abril de 2017 e entre os dias 01 e 10 de julho, foram selecionados, utilizando-se os critérios definidos na tabela 4, um total de 9. Estes resultados estão apresentados na tabela 6 .

Tabela 6 - Resultados de buscas para "gamification" "mathematics" "basic education"

\begin{tabular}{|l|l|}
\hline Google Scholar & 145 \\
\hline Scielo & 0 \\
\hline IEEE & 1 \\
\hline Totas de resultados & 146 \\
\hline Total de Selecionados & 9 \\
\hline
\end{tabular}

Fonte: Elaborada pelo autor.

Nesta etapa, somente $6,16 \%$ dos resultados obtidos foram selecionados após a análise, num total de 9 artigos/estudos.

Os critérios de exclusão mais utilizados foram:

- "3. Ensinos médio, superior e pós-graduações", com $66,44 \%$.

- $\quad$ "2. Outras áreas de ensino que não matemática" com $63,70 \%$.

- "4. Jogos não computacionais" com 27,40\%.

- $\quad$ "1. Jogos de entretenimento sem fins educativos" com 26,03\%.

(Obs. Alguns artigos foram eliminados por mais de um critério). A Figura 2 mostra os resultados.

Figura 2 - Critérios de exclusão mais utilizados para a busca "gamification" "mathematics" "basic education" 


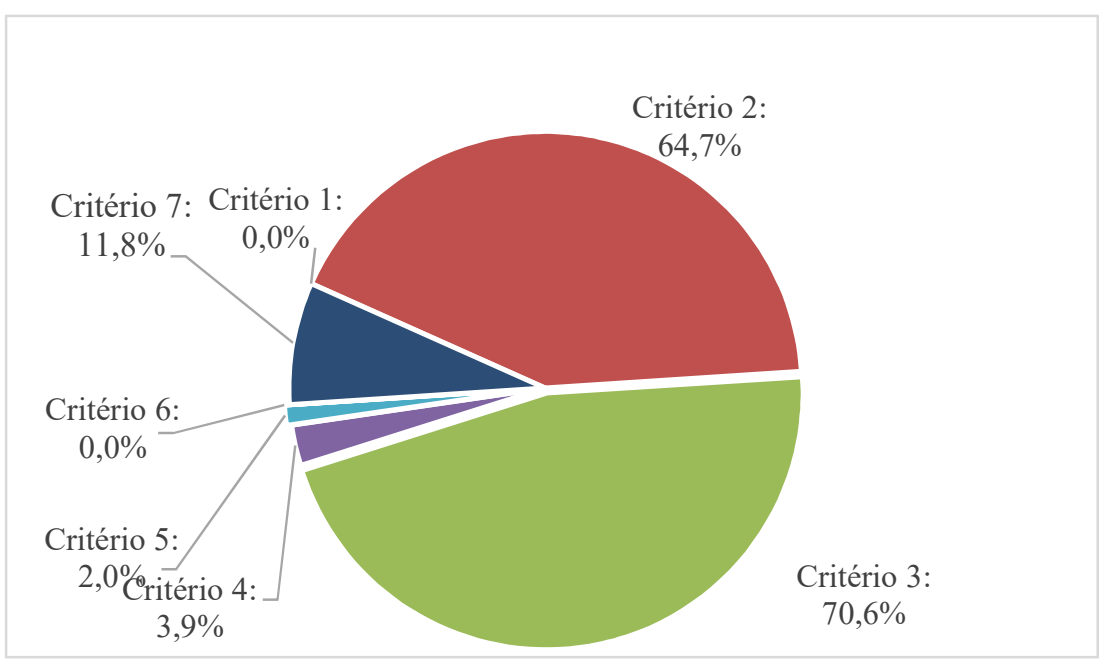

Ao final, a revisão sistemática resultou em 197 trabalhos, através das buscas nas bases escolhidas, sendo que 51 (Tabela 5) com a string \#1 e 146 (Tabela 6) com a string \#2. Destes, 15 foram selecionados (7,61\% do total de trabalhos encontrados na busca) após as etapas de seleção mencionadas anteriormente. A Figura 3 exibe o número de publicações selecionadas em cada ano. Embora consideremos um espaço de apenas 5 anos, parece haver um interesse crescente da comunidade científica pelo tema.

Figura 3 - Número de publicações selecionadas ao longo dos anos (2012-2017)

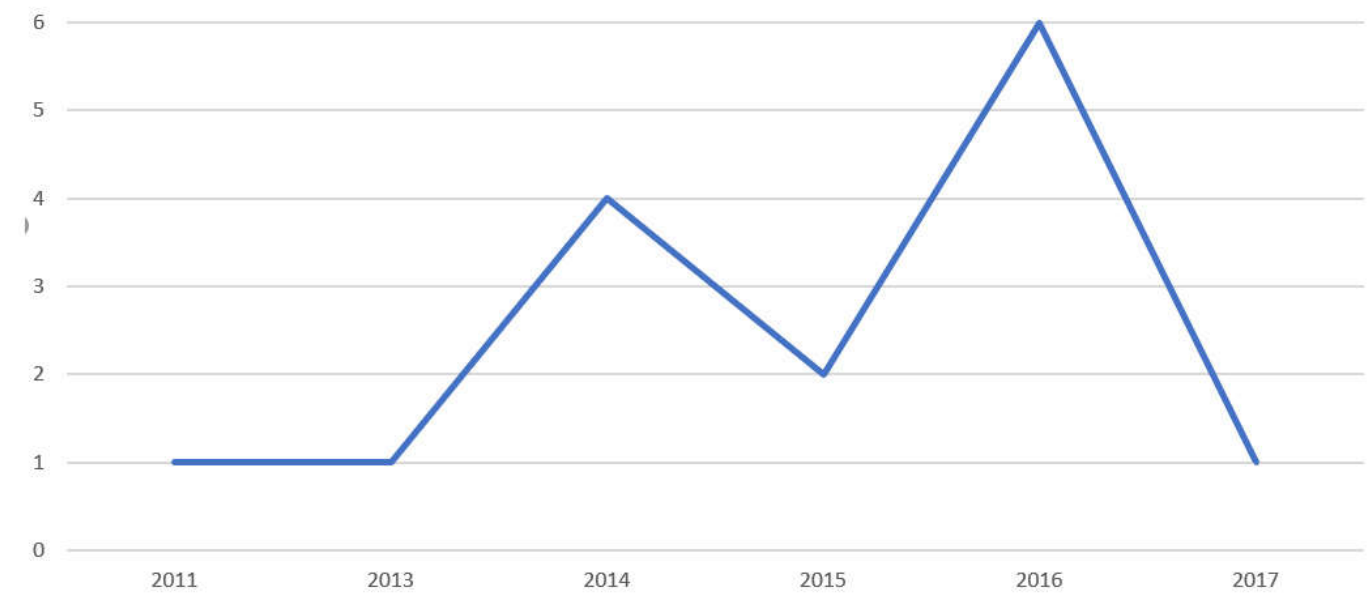

Separamos os artigos em três grandes grupos para uma melhor análise de suas características. O primeiro é de artigos que realizam somente revisões de literatura sobre o assunto, no total de 3 , o segundo reúne aqueles que relatam resultados de aplicações experimentais de jogos para o auxílio do processo de ensino-aprendizagem, em alguns casos descrevendo o jogo e seu processo de desenvolvimento, no total de 9 e, o último dos grupos reúne descrições do processo de desenvolvimento de jogos educativos, porém sem sua aplicação, no total de 3 . Os resultados finais estão apresentados na tabela 7 .

Tabela 7 - Resultados da pesquisa

\section{Artigos de Revisão de Literatura}


1. Mobile Gamification in Education Engage, Educate and Entertain via Gamified Mobile Apps | Autores: Ferial Khaddage, Christoph Lattemann, Ricardo AcostaDíaz - ShortURL: https://goo.gl/ADsSo7

- Explora a utilização de jogos em dispositivos móveis para educação.

2. The Question of Digital Game Based Learning: An Investigation into the Potential Promises and Perils of Education's Golden Goose | Autores: Molly E. O'Riley ShortURL: https://goo.gl/EgXjuq

- Artigo de investigação sobre o uso de games digitais na educação básica.

3. K-12 mobile learning | Autores: Cathy Cavanaugh, Dorit Maor, Aidan McCarthy ShortURL: https://goo.gl/BvfzXb

- Análise sobre o uso de dispositivos móveis para a educação básica.

Artigos que relatam resultados de aplicações experimentais

4. Aprender matemática jogando online? Levantamento e categorização de 100 jogos eletrônicos | Autores: Helder França Floret, Cleonice Puggian, Clícia Valladares Peixoto Friedmann - ShortURL: https://goo.gl/nFljV1

- Como jogos eletrônicos podem contribuir para o ensino da matemática na educação básica. Pesquisa exploratória com levantamento e classificação de jogos.

5. Serious Game Design Process, Study Case: Sixth Grade Math | Autores: Francisco J. Álvarez- Rodríguez, Arturo Barajas-Saavedra, Jaime Munoz-Arteaga - ShortURL: https://goo.gl/zkT1fo

- Busca definir bases para um processo de desenho de jogos sérios, fazendo um estudo de caso para um jogo da sexta série em matemática.

6. Juego serio como eje motivador en la asignatura de matemáticas de la unidad educativa héroes de Jambelí. | Autores: Castro Morocho, Rosa Annabel - ShortURL: https://goo.gl/oXRI9q

- Investiga a aplicação de jogos sérios realizado em uma escola de educação básica.

7. Developing Serious Games for 12-16 Year Old Students | Autores: Cecilia Sik Lanyi - ShortURL: https://goo.g1/UithHN

- Desenvolvimento de um jogo para ensino de matemática.

8. The Effects of Digital Games on Middle School Students' Mathematical Achievement | Autores: Pilar Lisa Starkey - ShortURL: https://goo.gl/5d4mnb

- Verificação dos efeitos de uso de games em educação básica de matemática.

9. Evaluating the impact of Mars and Venus Effect on the use of an Adaptive Learning Technology for Portuguese and Mathematics | Autores: Sivaldo J. de Santana, Ranilson Paiva, Ig Ibert Bittencourt - ShortURL: https://goo.gl/9mgyAf

- Avalia a efetividade do uso de plataformas digitais de ensino em estudantes do $5^{\circ}$ ao $9^{\circ}$ ano em matemática e português.

10. Sistema tutor afectivo para el aprendizaje de las matemáticas usando técnicas de gamificación | Autores: Ramón Zatarain-Cabada, María Lucia Barrón- Estrada, Jorge García-Lizárraga - ShortURL: https://goo.gl/1CpaUF

- Trata de um sistema que usa técnicas de gamificação para o ensino de matemática.

11. Mobile Learning and Mathematics -Foundations, Design, and Case Studies. Chapter 9 Using Mobile Games in the Classroom | Autores: Anders Kluge and Jan Dolonen - ShortURL: https://goo.gl/MT8oyp

SANTOS, S. L. T. dos; BÍSCARO, H. H. Revisão sistemática sobre a utilização de jogos sérios na aprendizagem de matemática. C.Q.D.- Revista Eletrônica Paulista de Matemática, Bauru, v. 14, p. 12-25, fev. 2019. Edição Ermac.

DOI: 10.21167/cqdvol14ermac201923169664sltshhb1225 Disponível em: https://www.fc.unesp.br/\#!/departamentos/matematica/revista-cqd/ 
- Investigação qualitativa e quantitativa sobre o uso do jogo Dragon- Box para o ensino de álgebra no ensino básico.

12. E-learning Sudan Final report Phase II | Autores: H.E. Stubbe, M. van der Klauw, J.J. Langefeld, N.C.M. Theu- nissen, A.H. van der Hulst - ShortURL: https://goo.gl/dNVh7j

- Mostra os resultados de uso de games para o ensino de matemática. cação

Artigos com descrições do processo de desenvolvimento de jogos, sem apli-

13. Proceso de Ensenanza-Aprendizaje enMatemáticas para el Diseno de Videojuegos Educativos | Autores: Jona- tan Belmontes Zacarias - ShortURL: https://goo.gl/7dHWzo

- Análise de resultados mexicanos sobre educação básica em matemática.

14. Jogo Digital para Motivar a Aprendizagem de Operações Aritméticas na Educação Básica | Autores: Leandro F. Mota, Edson P. Pimentel - ShortURL: https://goo.gl/nXqss7

- Discute o uso de jogos digitais como motivador para apoiar a aprendizagem de matemática.

15. Brincando com Matemática: uma alternativa educacional tangível e acessível ao ensino básico | Autores: Stéfanni Brasil da Silva, Ricardo Dalke Meucci - ShortURL: https://goo.gl/h6XFmG

- Desenvolvimento de jogo para ensino de conceitos básicos de matemática.

\section{Conclusão}

Esta sessão discorrerá sobre as principais conclusões obtidas através da análise sistemática de publicações científicas sobre a aplicação de jogos sérios digitais no ensino-aprendizagem de matemática.

Dentre os benefícios encontrados nos estudos analisados existem subsídios que mostram que o uso de jogos sérios pode auxiliar em uma das maiores dificuldades encontradas atualmente, a de manter o interesse do aluno em aprender. A introdução dos jogos, com sua dinâmica e contemporaneidade, é capaz de criar um ritmo que as aulas tradicionais não possuem, aumentando a motivação do aluno. O seu uso também resulta em crescimento de performance do processo de ensino-aprendizagem, melhorando os resultados da construção de conhecimentos da matemática. (ÁLVAREZ-RODRÍGUEZ; BARAJAS-SAAVEDRA; MUÑOZ-ARTEAGA, 2014; CASTRO-MOROCHO; JUMBO CASTILLO, 2016; SANTANA et al, 2016; STARKEY, 2013; ZATARAIN-CABADA; BARRÓN-ESTRADA; GARCÍA-LIZÁRRAGA, 2016)

Sua utilização possibilita também o acesso a esta educação em localidades mais remotas, onde muitas vezes, por escassez de recursos financeiros dos governos e consequentemente há pouca oportunidade de aprendizagem para os estudantes. Os jogos sérios, e os sistemas de tutoria "gamificados" são auxílios importantes em localidades onde existe carência de mão de obra qualificada, influindo beneficamente, ocasionando um aumento na qualidade da educação (SANTANA et al, 2016; STUBBÉ, 2016; ZATARAIN-CABADA; BARRÓN-ESTRADA; GARCÍA-LIZÁRRAGA, 2016)

O aprendizado de matemática, estimulado pelo uso de jogos sérios, muda a atitude dos alunos frente à disciplina, tida muitas vezes como muito difícil, um "pesadelo estudantil", criando inclusive um aumento na autoestima das crianças por conseguirem compreender conceitos da matéria e resolverem exercícios (STUBBÉ, 2016). Papert (1993 apud SILVA; MEUCCI, 2017) 
dá o nome de "Matofobia" a este medo de matemática, que leva a uma falsa crença que há estudantes privilegiados quanto à compreensão de matérias de exatas.

Uma das maiores dificuldades apontadas para a realidade brasileira, é a pouca disponibilidade de títulos em língua nativa para o ensino de matemática dentre os disponíveis gratuitamente, estudados e catalogados (FLORET; PUGGIAN; FRIEDMANN, 2016). Porém existem iniciativas promissoras através do uso de sistemas de tutoria "gamificados" que podem contribuir muito na aprendizagem (SANTANA et al, 2016), no desenvolvimento de jogos para motivar a aprendizagem matemática (MOTA; PIMENTEL, 2014), e para a utilização em casos de educação inclusiva (SILVA; MEUCCI, 2017).

Potenciais problemas encontrados dizem respeito à inadequação dos jogos à faixa etária $\mathrm{e}$ ao conhecimento a ser transmitido, o que pode apresentar grandes variações na melhora do aprendizado (LANYI, 2011). O mesmo pode ocorrer no caso de alunos dos sexos masculinos e femininos, dependendo do tema do jogo sério utilizado (SANTANA et al, 2016; STARKEY, 2013).

Apesar de uma das características dos jogos sérios preconizar a autonomia no uso e na aprendizagem, intervenções de um instrutor, relacionando conceitos dos jogos com a grade curricular, resulta em melhor rendimento dos alunos nas avaliações, mostrando a necessidade de uma abordagem pedagógica bem definida na utilização destes jogos sérios eletrônicos, para alavancar o processo de ensino-aprendizagem (KLUGE; DOLONEN, 2015).

Os resultados obtidos apontam que a utilização de jogos sérios eletrônicos é efetiva para a melhoria do processo de ensino-aprendizagem da matemática. Entretanto, existem diversos aspectos a serem considerados em sua utilização, como a correta contextualização do público alvo, o uso de técnicas pedagógicas que apoiem o uso dos jogos, o relacionamento entre o que foi aprendido no jogo com os conceitos matemáticos formais, e a capacitação dos professores para a utilização desta tecnologia.

Sumarizando, a revisão realizada indica um grande interesse da comunidade científica sobre o tema. Além disso, jogos sérios são uma ferramenta com um potencial apreciável, porém, a sua aplicação deve ser uma atividade bem planejada, com objetivos claros e bem delineados para que seu potencial de ensino-aprendizagem possa ser plenamente aproveitado pelos educadores, ou seja, apesar de ser caráter lúdico, essa não deve ser uma atividade meramente recreativa.

Uma razão possível para o potencial da aplicação de jogos sérios é a grande familiaridade com que os estudantes contemporâneos se relacionam com o universo digital. Poder-se-ia dizer que eles apresentam uma predisposição "natural" para o aprendizado das habilidades cognitivas necessárias para um bom desempenho nos jogos. Some-se a isso a natureza competitiva inerente a todos os seres humanos e teremos uma boa ferramenta de ensino-aprendizagem em qualquer campo do conhecimento, particularmente na Matemática, que é uma área considerada árdua pela maioria dos estudantes.

\section{Referências}

ÁLVAREZ-RODRÍGUEZ, F. J.; BARAJAS-SAAVEDRA, A.; MUÑOZ-ARTEAGA, J. Serious game design process, study case: sixth grade math. Creative Education, v. 05, Scientific Research Publishing, Inc., n. 09, p. 647-656, 05 2014. Disponível em: $<$ https://goo.gl/p1JuU4>. Acesso em: 1 nov. 2017.

BECK, J. C.; WADE, M. The kids are alright: how the gamer generation is changing the workplace. Boston: Harvard Business Review Press, 2006.

SANTOS, S. L. T. dos; BÍSCARO, H. H. Revisão sistemática sobre a utilização de jogos sérios na aprendizagem de matemática. C.Q.D.- Revista Eletrônica Paulista de Matemática, Bauru, v. 14, p. 12-25, fev. 2019. Edição Ermac.

DOI: 10.21167/cqdvol14ermac201923169664sltshhb1225 Disponível em: https://www.fc.unesp.br/\#!/departamentos/matematica/revista-cqd/ 
BIOLCHINI, J. et. al. Systematic review in software engineering. Rio de Janeiro, 2005.

CASTRO MOROCHO, R. A.; JUMBO CASTILLO, E. M. Juego serio como eje motivador en la asignatura de matemáticas de la unidad educativa héroes de Jambelí. $158 \mathrm{f}$. Trabajo de Pregrado (Carrera de Comunicación Social) -Universidad Tecnica de Machala, Machala, 2016. Disponível em: <https://goo.gl/2bghjq>. Acesso em: 1 nov. 2017.

DETERDING, S. et. al. Gamification. using game design elements in non-gaming contexts. In: CHI CONFERENCE ON HUMAN FACTORS IN COMPUTING SYSTEMS, 11., 2011, Vancouver. Proceedings... Vancouver: Acm, 2011. p. 2425 - 2428. Disponível em:

$<$ https://goo.gl/a9rwDH>. Acesso em: 5 out. 2017.

DJAOUTI, D.; ALVAREZ, J.; JESSEL, J.-P. Classifying serious games: the g/p/s model. In: FELICIA, P. (Ed.). Handbook of research on improving learning and motivation through educational games: multidisciplinary approaches. Hershey: Igi Global, 2011. p. 118-136. Disponível em: <https://goo.gl/ivP8wh>. Acesso em: 17 out. 2017.

FLORET, H. F.; PUGGIAN, C.; FRIEDMANN, C. V. P. Aprender matemática jogando online? Levantamento e categorização de 100 jogos eletrônicos. Almanaque Multidisciplinar de Pesquisa, v. 1, n. 2, p. 4-17, ago. 2016. Disponível em: $<$ https://goo.gl/jv6uW8>. Acesso em: 1 nov. 2017.

FREIRE, P. Pedagogia do oprimido. 17. ed. Rio de Janeiro: Paz e Terra, 1987.

KITCHENHAM, B. Procedures for performing systematic reviews, Technical Report TR/SE-0401. Department of Computer Science, Keele University and National ICT. Australia. 2004.

KLUGE, A.; DOLONEN, J. Using mobile games in the classroom: the good and the bad of a new math language. In: CROMPTON, H.; TRAXLER, J. (Ed.). Mobile learning and mathematics: foundations, design, and case studies. New York: Routledge, 2015. p.106-121.

LANYI, C. S. Developing serious games for 12-16 year old students. In: INFORMING SCIENCE \& IT EDUCATION CONFERENCE (INSITE), 11., 2011, Novi Sad. Proceedings... Santa Rosa: Informing Science Institute, 2011. p. 521-535. Disponível em: $<$ http://proceedings.informingscience.org/InSITE2011/InSITE11p521-535SikLanyi333.pdf $>$. Acesso em: 1 nov. 2017.

MALLETT, R. et. al. The benefits and challenges of using systematic reviews in international development research. Journal of Development Effectiveness, v. 4, n. 3, p. 445-455, set. 2012. Disponível em: <https://goo.gl/YxKrgS>. Acesso em: 1 nov. 2017.

MOTA, L. F.; PIMENTEL, E. P. Jogo digital para motivar a aprendizagem de operações aritméticas na educação básica. In: CONFERÊNCIA LATINO-AMERICANA DE OBJETOS E

SANTOS, S. L. T. dos; BÍSCARO, H. H. Revisão sistemática sobre a utilização de jogos sérios na aprendizagem de matemática. C.Q.D.- Revista Eletrônica Paulista de Matemática, Bauru, v. 14, p. 12-25, fev. 2019. Edição Ermac.

DOI: 10.21167/cqdvol14ermac201923169664sltshhb1225 Disponível em: https://www.fc.unesp.br/\#!/departamentos/matematica/revista-cqd/ 
TECNOLOGIAS DE APRENDIZAGEM, 9., 2014, Manizales. Proceedings... Manizales: Laclo, 2014. p. 155-165. Disponível em: $<$ https://www.researchgate.net/profile/Edson_Pimentel/publication/278158060_Jogo_Digital_para_Motivar_a_Aprendizagem_de_Operacoes_Aritmeticas_na_Educacao_Basica/links/557c8dae08aeb61eae2363bf.pdf $>$. Acesso em: 21 jan. 2019.

NIGHTINGALE, A. A guide to systematic literature reviews. Surgery (Oxford), v. 27, n. 9, p. 381-384, set. 2009. Disponível em: <https://goo.gl/RqpTVk>. Acesso em: 30 out. 2017.

NUNES, F. L. Revisão Sistemática. 2010. Slides em PDF utilizados na disciplina Metodologia da Pesquisa em Sistemas de Informação, parte do Programa de Pós-graduação em Sistemas de Informação da EACH-USP.

PALFREY, J.; GASSER, U. Nascidos na era digital: entendendo a primeira geração de nativos digitais. Porto Alegre: Artmed, 2011.

SANTANA, S. J. de et. al. Evaluating the impact of mars and venus effect on the use of an adaptive learning technology for portuguese and mathematics. In: IEEE INTERNATIONAL CONFERENCE ON ADVANCED LEARNING TECHNOLOGIES (ICALT)., 2016, Austin. Proceedings... Los Amitos: Cps Conference Publishing Services, 2016. p. 31 - 35. Disponível em: <https://goo.gl/itmzBV>. Acesso em: 1 nov. 2017.

SILVA, S. B. da; MEUCCI, R. D. Brincando com matemática: uma alternativa educacional tangível e acessível ao ensino básico. Iniciação: Revista de Iniciação Científica, Tecnológica e Artística, v. 6, n. 4, p. 14-21, abr. 2017. Disponível em: <https://goo.g1/fXQ38H>. Acesso em: 1 nov. 2017.

STARKEY, P. L. The effects of digital games on middle school students' mathematical achievement. 2013. $135 \mathrm{f} \mathrm{p}$. Theses (Doctor of Education) - Lehigh University, Bethlehem, 2013. Disponível em: <https://goo.gl/2XnU62>. Acesso em: 1 nov. 2017.

STUBBÉ, H. E. et. al. E-learning Sudan final report phase II. Soesterberg, 2016. Disponível em: <https://goo.gl/bwZeXH>. Acesso em: 6 out. 2017.

TAMÁSSIA DOS SANTOS, S. L.; BISCARO, H. H. Utilização de jogos sérios na aprendizagem de matemática: Uma revisão sistemática. In: ENCONTRO REGIONAL DE MATEMÁTICA APLICADA E COMPUTACIONAL, 5., 2018, Bauru. Caderno de trabalhos completos e resumos... Bauru: Unesp, 2018. p. 373-379. Disponível em: $<$ http://www.fc.unesp.br/\#!/departamentos/matematica/eventos2341/ermac-2018/>. Acesso em: 30 jul. 2018.

ZATARAIN-CABADA, R.; BARRÓN-ESTRADA, M. L.; GARCÍA-LIZÁRRAGA, J. Sistema tutor afectivo para el aprendizaje de las matemáticas usando técnicas de gamificación. Research In Computing Science: An open access research journal on 
Computer science and computer engineering, v. 111, n. 1, p. 83-96, Sept. 2016. Disponível em: <https://goo.gl/8u3hCx>. Acesso em: 19 nov. 2017. 